\title{
La réinvention de Rimbaud par la poésie chypriote
}

\section{Yiannis E. IOANNOU}

\section{(2) OpenEdition}

\section{Journals}

Édition électronique

URL : http://journals.openedition.org/transtexts/96

DOI : 10.4000/transtexts.96

ISSN : 2105-2549

\section{Éditeur}

Gregory B. Lee

\section{Édition imprimée}

Date de publication : 1 janvier 2007

Pagination : 183-189

ISSN : 1771-2084

\section{Référence électronique}

Yiannis E. IOANNOU, «La réinvention de Rimbaud par la poésie chypriote », Transtext(e)s Transcultures 跨文本跨文化 [En ligne], 2 | 2007, mis en ligne le 11 juin 2009, consulté le 10 décembre 2020. URL: http://journals.openedition.org/transtexts/96 ; DOI : https://doi.org/10.4000/transtexts.96 


\title{
La réinvention de Rimbaud par la poésie chypriote
}

\author{
YIANNIS E. IOANNOU
}

This article studies the reception of Rimbaud's two-period stay in Cyprus (1878-79, $1880)$, by the $20^{\text {th }}$ century Cypriot poetry. It attempts to show that, despite the fact that Rimbaud's activities during his stay, had nothing to do with literature or anything heroic, Cypriot poetry has presented the poet as a semaphore of individual and national revolution as well as an idealised example of contestation and rebelliousness.

Les relations historiques attestées entre Chypre et la France remontent à 1192, date à laquelle la dynastie des Lusignans s'installe dans l'île pour y régner jusqu'en 1489. Malgré l'hellénisation progressive des descendants des Lusignans-dont le roi Pierre I (1359-1369) en constitue un illustre exemple-les Chypriotes avaient emprunté une foule d'éléments linguistiques au français de cette période. ${ }^{1}$ Aussi, le dialecte chypriote conserve jusqu'à nos jours bon nombre de mots français adaptés

\footnotetext{
${ }^{1}$ Voir à ce propos, la langue employée par Leontios Machairas dans sa célèbre Chronique datant du XVe siècle, dans Sylvain Béraud, La culture française dans l'espace chypriote, Nicosie, Publication du Service Culturel du Ministère de l'Éducation de Chypre, 1990, 75, pp. 78-83. Si Béraud peut en inférer (p.78) que « sur les cent soixante mots repérés, une dizaine, aujourd'hui, demeurent encore en usage », il convient de nuancer ce recensement qui ne reflète pas la réalité. Dans le parler rural en effet, un nombre important de mots d'origine française demeure encore en usage, ce qui laisse présumer que le volume

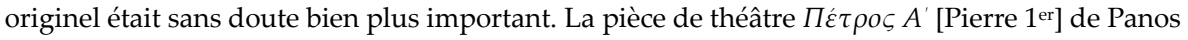
Ioannidès, Nicosie 1991, constitue une très louable tentative de restitution de la langue de l'époque des Lusignans.
} 
dans leur majorité écrasante, au système linguistique chypriote. Nous devons noter que ces termes, le plus souvent inconnus du grec moderne «standard » d'Athènes, véhiculent une authenticité chypriote profonde et marquent souvent la différence entre le parler citadin qui tend à les évacuer et celui des campagnes qui non seulement les conserve mais les maintient de surcroît en usage. Pour n'en citer que

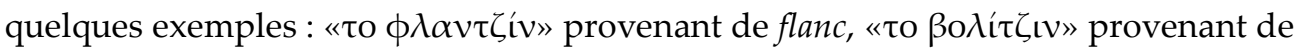
volige, « $\eta \tau \sigma \alpha ́ \mu \pi \rho \alpha-\eta \tau \sigma \alpha \mu \pi \rho o u ́$ provenant de chambre appartiennent presque exclusivement au registre rural et sont une marque d'une identité strictement chypriote par opposition au restant de l'hellénisme. ${ }^{2}$

Il est vrai que la période ottomane ne compte pas parmi les plus fécondes en ce qui concerne non seulement la francophonie mais plus généralement les langues et la culture. $^{3}$ En dépit de ce que les voyages à Chypre de Lamartine, Rimbaud et autres hommes de lettres français pourraient laisser entendre. Les deux séjours effectués par Arthur Rimbaud (1878-79 et 1880), en dépit du caractère prosaïque et anecdotique des lettres adressées aux siens qui en sont apparemment le seul témoignage, ont toutefois acquis une dimension symbolique et se sont incorporés à la mémoire intellectuelle collective de Chypre, excitant l'imagination des créateurs chypriotes. D'après ces lettres, les deux séjours du poète se présentent plutôt comme un choix forcé qui consiste à satisfaire les besoins de survie du poète. Rien ne rappelle l' image idyllique de " l'île d'Aphrodite » (Ronsard), de « l'île du vin et de la beauté » (Baudelaire) telle qu'on la retrouve dans la littérature française de la période entre le Moyen-Age et le mouvement romantique du XIXe siècle. ${ }^{4} \mathrm{Au}$ contraire, Rimbaud insiste sur le caractère désertique, sur le malaise provoqué par les moustiques et les maladies et sur le comportement rustre des ouvriers locaux. Cette image quasi sinistre de l'île et de ses habitants aurait dû, normalement, provoquer une réaction négative de la part des intellectuels de l'île, en général très sensibles à l'image que les étrangers ont de leur pays, dans la mesure où elle ne flatte pas la vanité des Chypriotes. Malgré cela, nous nous trouvons devant un paradoxe puisque, comme le dit la poétesse Pitsa Galazi:

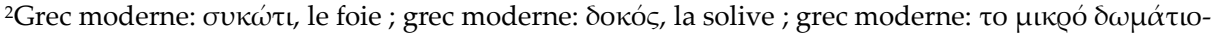

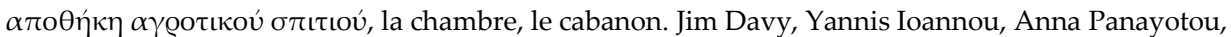
«French and English loans in Cypriot diglossia », in Chypre hier et aujourd'hui, Travaux de la Maison de l'Orient Méditerranéen, Numéro 25, Lyon 1996, pp. 127-136.

${ }^{3}$ Voir Béraud, La culture française dans l'espace chypriote. Voir également Paul et Anna Pouradier Duteil, Chypre au temps de la révolution Française, Nicosie, Publication du Ministère de l'Éducation de Chypre, 1989; Pitsa Galazi, Le bel Arthur ou Arthur Rimbaud à l'île de Chypre, Nicosie, Éditions Onissilos, 1991. ${ }^{4}$ Yiannis E. Ioannou, «La présence de Chypre dans la poésie française », Revue Intertextes, Numéro 3, Salonique 2001, pp. 149- 156.
} 
Par les vers remarquablement traduits, le poète français a été d'une importance capitale dans la formation des poètes de la Grèce métropole. Avec eux, les poètes de la génération chypriote antérieure, comme Vrahimis, Valdasséridis (dans une moindre mesure), Xanthos Lyssiotis et Manos Kralisqui récitaient de mémoire de longs extraits des poèmes de Rimbaud- ont été tributaires de son influence. ${ }^{5}$

Nous allons donc tenter de voir de plus près la réception de Rimbaud par la littérature chypriote. Et plus particulièrement, le poète Manos Kralis, considéré comme un des poètes maudits de la poésie chypriote qui avait intitulé un de ses recueils, Un automne en enfer, titre qui renvoie à l'œuvre de Rimbaud, Une saison en enfer. ${ }^{6} \mathrm{D}^{\prime}$ après certains critiques, des expériences similaires comme la perte de leur père à un âge précoce, leur santé problématique et l'atmosphère de l'hôpital, le goût de la quête et de l'aventure, constitueraient des âxes communs aux deux poètes:

Kralis, en raison des expériences similaires à celles de Rimbaud, se considérait, lui aussi, comme un «maudit » dans la vie et il l'avait imité en littérature. ${ }^{7}$

Le rôle novateur de l'œuvre rimbaldienne sur l'échelle non seulement française mais également mondiale se retrouve chez Kralis par rapport à la poésie chypriote, même avec quelques décennies de retard. En effet, le poète chypriote est considéré comme celui qui s'oppose à l'ordre établi, qui stigmatise le mode de vie petit bourgeois et qui introduit l'écriture poétique moderne dans la poésie chypriote. ${ }^{8}$ Ainsi Rimbaud a-t-il pu être désormais perçu comme l'éphèbe rebelle, l'aventurier, aussi insoumis que le seront les héros de l'indépendance chypriote soixante-dix ans plus tard. La poétesse Pitsa Galazi établit cet insolite parallélisme en ces termes:

Rimbaud le subversif et le révolutionnaire touche, de manière essentielle, directe et dynamique Chypre par le biais des traductions vers 1960. Cette date situe aussi la naissance de la poésie de ma propre génération. Une époque qui trouve Chypre encore toute chaude de la lutte pour son indépendance et de laquelle elle vient de sortir. Ainsi donc, paradoxalement - et c'est bien connu, en poésie, tout paradoxe ou toute concoction poétique et associations curieuses sont permis-, la révolution de Rimbaud se joint à la nôtre. Par réaction, peutêtre, au silence imposé par la censure britannique, nous avons démoli ses limites en présentant de la poésie. Peut-être aussi parce que notre lutte comportait un contenu spirituel plus profond. Peut-être encore parce que les

5Pitsa Galazi, «Arthur Rimbaud, sémaphore de la poésie chypriote », in Arthur Rimbaud ou le voyage poétique, Collection In-texte, Toulouse, Tallandier, 1992, p. 62.

${ }^{6}$ Costas Papayiannidis, Manos Kralis (1914-1989) et le renouveau du discours poétique à Chypre, Services Culturels du Ministère de l'Éducation et de la Culture, Collection Thèses de Doctorat, Numero 6, Nicosie ,2004, pp. 140-146.

${ }^{7}$ Papayiannidis, pp. 141-142 et p. 146.

8Papayiannidis, pp. 143-144. 
enfants héroïques de Chypre de ces années-là étaient du même âge que Rimbaud, et écrivaient de la poésie ou étaient déjà poètes et s'acheminaient vers le sacrifice parce qu'ils avaient adoré et avaient réclamé leur liberté. Je ne sais pas vraiment. En apparence Rimbaud n'avait rien à voir et pourtant, de manière inexpliquée, il y avait un rapport et il devint ferment de la révolution au fond de la voix poétique du pays. ${ }^{9}$

Et si Chypre est comparée à un «bateau ivre » par la romancière grecque Tatiana Gritsi-Milliex dans sa Préface du recueil de Pitsa Galazi (1991), c'est aussi parce que « le jeune Eole des Illuminations » est dans ce recueil mis en parallèle avec les héros de l'Indépendance chypriote (1955-59). ${ }^{10}$ Voici un extrait du poème tel qu'il a été publié dans L'Ivre Caravane:

Le fleuve a été coupé en deux Jean-Baptiste avance à travers Sa tête sous les bras Il marche sur les vagues Ouvrant les routes du Paradis Comptant les jours de l'herbe sur les doigts Se réconciliant avec les sauterelles Il prophétise dans l'éternité d'une voix fertile qui a tiré toute sa force des entrailles et déborde d'enfants comme le fleuve de ma terre et de ma génération

Siffle le jeune Eole des Illuminations léguant le bateau ivre de ma nostalgie là ou se brisent les polyèdres du sommeil et d'où Lazare dissimulé soufflait dans mes baudruches de couleur pour qu'elles crèvent stimulant mon exil et mon bannissement ${ }^{11}$

Le fait que certains des jeunes maquisards de la révolte de 1955-59 contre les Britanniques étaient des poètes visionnaires- en particulier Evagoras Pallikaridis pendu par les Britanniques à l'âge de 18 ans, le 14 mars 1957, - ne pouvait que

\footnotetext{
9Papayiannidis, p. 63.

${ }^{10}$ Tatiana Gritsi-Milliex, épouse de Roger Milliex, est considérée comme la représentante du Nouveau Roman en Grèce. Galazi, « Arthur Rimbaud, sémaphore de la poésie chypriote », p. 13.

${ }^{11}$ Lefkios Zafiriou, L'Ivre Caravane, Paris, Éditions Bleu Outremer,1993, pp. 18-19. Traduction française effectuée par l'auteur du présent article.
} 
renforcer le parallélisme entre le personnage et la révolte de Rimbaud et les héros de l'indépendance chypriote. ${ }^{12}$

Un peu plus tard, le poète Lefkios Zafiriou, qui a lui aussi été marqué par le caractère emblématique de la vie de Rimbaud, identifie la révolte rimbaldienne avec la révolte du peuple chinois et le transforme en un fougueux cycliste, témoignant à la fois du développement frénétique du tourisme chypriote et de la révolte du peuple chinois sur la place de Tiananmen..$^{13} \mathrm{~S}^{\prime}$ inspirant des lettres que Rimbaud avait envoyées de Chypre, il transgresse les limites spatio-temporelles afin de se replacer dans le contexte contemporain et de discrètement critiquer le développement effréné du tourisme dans cette ville de Limassol d'où Rimbaud postait ses lettres lorsqu'il résidait dans le mont Troodos. Voici le poème:
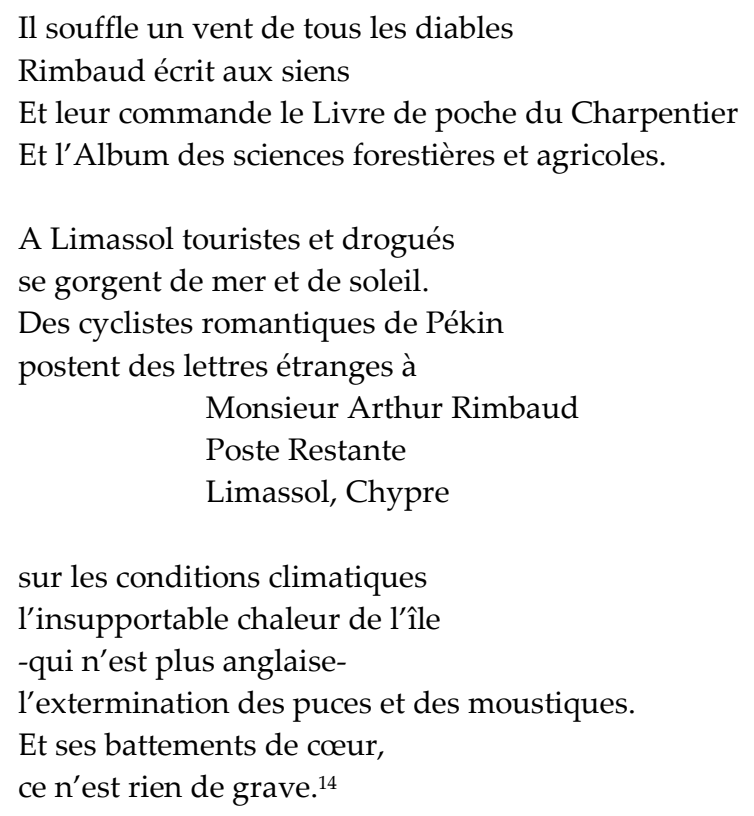

Nous devons également mentionner le fait que la présence de Rimbaud a inspiré des poètes populaires, entrant dans la légende locale. ${ }^{15}$ Le poète serait ainsi tombé gravement malade (fait en soi nullement improbable, si l'on se réfère à ses lettres) et

\footnotetext{
${ }^{12}$ Voir également à ce sujet, La lettre de protestation publiée par Albert Camus en décembre 1955 dans L'Express, à propos de la condamnation à mort par pendaison le 28 octobre 1955 d'un autre jeune maquisard, de Michel Caraolis, finalement pendu le 10 octobre 1956.

13Zafiriou, L'Ivre Caravane.

${ }^{14}$ Zafiriou, pp. 20-21.

${ }^{15}$ Zafiriou, p. 24.
} 
il se serait fait soigner par une très pauvre famille de villageois de Xylofagou, au sud-est de l'île. Toujours selon cette tradition, il portait dans son sac une somme importante d'argent qui correspondait aux salaires des ouvriers de la carrière qu'il n'avait pas eu le temps de payer. Après être resté couché, à moitié inconscient pendant trois jours, il avait fini par reprendre connaissance. Cherchant sa sacoche du regard, il avait trouvé l'argent intact! Le poète populaire Petros Evangelou «qui nous accueillit à l'entrée du village de Xilophagou, à Chypre, avec une parole spontanée, mémoire renouvelée d'un autre accueil, celui que son aïeule fit à Arthur Rimbaud malade, et qu'elle nourrit et réconforta », récite en vers et dans le dialecte chypriote l'histoire rapportée de bouche en bouche. ${ }^{16}$ Le vieil Andriotis- c'était le nom du villageois qui aurait accueilli Rimbaud -était l'arrière-grand-père du poète populaire qui nous rapporte l'incident avec une fierté évidente. Voici un extrait de ce texte dialectal, dans notre traduction:

...Cela était un grand honneur pour tout notre village
Comme cela l'était aussi pour tout notre lignage
Qu'il était poète, on ne l'a su qu'après
De sorte que le peuple l'a d'autant plus apprécié
C'était Arthur Rimbaud, le grand poète français
Qui écrivit plus de poésie qu'aucun autre n'a fait.
Et moi vrai descendant de ceux qui l'ont secouru
De leur comportement suis très fièrement ému. ${ }^{17}$
......

Ainsi donc, même si la Chypre de Rimbaud n'était à l'époque «qu'un chaos de rocs.../...Pas de terre, pas de jardins, pas un arbre...Les puces sont un supplice affreux,...», et même si lui-même n'avait vu que les aspects négatifs de ces séjours, sa figure exemplaire et la présence poétique de cet éternel adolescent ont fait vibrer une corde poétique particulière. ${ }^{18}$ Le passage de Rimbaud sur l'île à l'époque où les Britanniques s'installaient aussi, n'allait plus passer dans les écrits du poète français

\footnotetext{
${ }^{16}$ Zafiriou, L'Ivre Caravane, p. 5, p. 16 et p. 24. C'est Roger Milliex qui avait identifié la carrière où Rimbaud avait travaillé lors de son premier séjour, comme étant celle située près de Potamos (Liopétri), entre Xilophagou et Ayia Napa. Selon une autre opinion, elle serait située près du village d'Oroklini au nord de Larnaca. Voir à ce sujet Claude Legagneux, " Rimbaud in Cyprus or an episode of the tragic life of a poet ", Cyprus Review, octobre 1954, pp. 10-12. Achilléas Lymbouridis reprend cette thèse dans

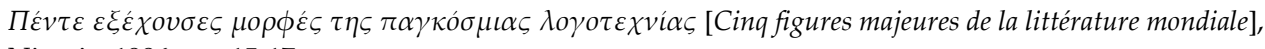
Nicosie, 1996, pp. 15-17.

${ }^{17}$ Zafiriou, L'Ivre Caravane, p. 24. Le texte de P. Evangélou n'a malheureusement pas été corrigé par un connaisseur du dialecte chypriote. Aussi, les fautes empêchent souvent la compréhension du texte. ${ }^{18}$ Arthur Rimbaud, CEuvres Complètes-Correspondance, Paris, Éditions Laffont, 2004: Lettres du 15 février 1879 et du 24 avril 1879, pp. 258-259.
} 
sachant que celui-ci avait déjà abandonné ses ambitions littéraires, mais de façon paradoxale et même surprenante, il est passé dans la littérature chypriote comme sémaphore de la révolte individuelle et nationale, comme un exemple idéalisé de contestation et d'insoumission, enfin, comme un chasseur de rêve, un décrypteur de l'autre vie qui ne cesse d'alimenter la verve poétique si fortement installée sur l'île, depuis déjà trois millénaires. 